www.jmscr.igmpublication.org

Impact Factor 5.84

Index Copernicus Value: 71.58

ISSN (e)-2347-176x ISSN (p) 2455-0450

crossref DOI: _https://dx.doi.org/10.18535/jmscr/v5i12.73

Journal Of Medical Science And Clinical Research

\title{
A Comparative Study of Glycated Albumin versus Glycated Haemoglobin in Diabetic Patients with End Stage Renal Disease on Haemodialysis
}

\author{
Authors \\ Dr Basanth Kumar ${ }^{1}$, Dr Megha Reddy ${ }^{2}$
}

${ }^{1}$ Associate Professor, Dept of General Medicine, Kempegowda Institute of Medical Sciences, Bangalore

${ }^{2}$ Senior Resident, Department of General Medicine, M S Ramaiah Medical College, Bangalore

\begin{abstract}
Objective: to compare glycated albumin $(G A)$ and glycated haemoglobin(HbAlC) as glycemic indicator in diabetic patients with End stage renal disease(ESRD) on haemodialysis (HD)and to assess which of the two parameters provides more accurate assessment of glycemic control.
\end{abstract}

Methodology: This is study which included 100 patients, 50 cases of diabetic ESRD patients on HD, and 50 controls of diabetic patients with normal renal function, from both the out-patient and in-patient Department of General Medicine, Kempegowda Institute of Medical Sciences, Bangalore between January 2016 to June 2016. HbAlC and Glycated Albumin was compared between these groups.

Results: FBS, PPBS and GA in controls was $187 \pm 57.8 \mathrm{mg} / \mathrm{dl}, 231.7 \pm 73.7 \mathrm{mg} / \mathrm{dl}, 72.84 \pm 32.42 \mathrm{ng} / \mathrm{ml}$ and in cases was $184 \pm 53.1 \mathrm{mg} / \mathrm{dl}, 224.7 \pm 72.5 \mathrm{mg} / \mathrm{dl}, 75.20 \pm 34.7 \mathrm{ng} / \mathrm{ml}$ respectively. The difference of $F B S$, PPBS and GA between controls and cases was not statistically significant. However, HbAlc was significantly lower in cases $(7.08 \pm 1.2)$ compared to controls $(9.26 \pm 2.01)$

Conclusion: The HbAlc levels were significantly and paradoxically lower in diabetic patients with ESRD on HD compared to diabetics with normal renal function. GA better reflected the glycemic control in these patients.

Keywords: Diabetes Mellitus, End stage renal disease, HbA1C, Glycated Albumin, Haemodialysis.

\section{Introduction}

Strict glycemic control in patients with diabetes decreases the incidence of diabetic complications ${ }^{1}$, which can determine the quality of life and prognosis of such patients. Intensive treatment with insulin or oral hypoglycaemic agents (OHAs) has been established to delay the onset and slow the progression of diabetic microangiopathy in the patients with types 1 diabetes and type 2 diabetes in the Diabetes Control and Complications Trial ${ }^{2}$ and the Kumamoto Study ${ }^{3}$.

Furthermore, a reduction of the risk for the development of diabetic microangiopathy in patients with type 2 diabetes by strict glycemic control was demonstrated in the UK Prospective Diabetes Study ${ }^{4}$. Recent clinical evidence has suggested the favorable effects of strict glycemic control on cardiovascular disease, a main cause of death in patients with diabetes. 5

Several clinical tests are useful for measuring long-term glycemic control in the general diabetic population. These same tests are routinely performed in diabetic subjects with chronic kidney disease (CKD) and end-stage renal disease (ESRD); however, their accuracy in these patients has not been rigorously tested. 
Hemoglobin A1c (HbA1c), the most widely used assay, measures the percentage of circulating haemoglobin $(\mathrm{Hb})$ that has chemically reacted with glucose and reflects ambient blood glucose control over the prior 120 days, with the most profound effect in the preceding 30 days. ${ }^{7,8}$

Factors that shorten red blood cell (RBC) survival, including severe nephropathy, may reduce $\mathrm{HbA} 1 \mathrm{c}$ since the time necessary for glucose to chemically bond with RBCs decreases. ${ }^{9}$ If this significantly impacts $\mathrm{HbA1c}$, dialysis patients and clinicians would be falsely comforted by relatively low HbA1c values despite high risk for subsequent cardiovascular disease and infectious complications.

In recent studies, serum glycated albumin (GA) has been hypothesized to be an alternative marker for glycemic control in patients with diabetes, which is not affected by changes in the survival time of erythrocytes. They evaluated diabetic HD patients and diabetic subjects without nephropathy and demonstrated that HbA1c severely underestimates true glucose control relative to the GA assay. ${ }^{10,11}$

Therefore, the present study was designed to assess whether GA might provide a better indicator than HbA1c for glycemic control in HD patients with diabetes.

In a study by Inaba et al. (Japan , 2007) composed of 538 HD patients with type 2 diabetes, 828 HD patients without diabetes, and 365 patients with type 2 diabetes and normal renal function, it was concluded that GA provides a significantly better measure to estimate glycemic control in HD patients with diabetes and that the assessment of glycemic control by HbA1c in these patients might lead to underestimation likely as a result of the increasing proportion of young erythrocyte by the use of erythropoietin. ${ }^{11}$

In a study by Peacock et al. (USA, 2008) HbA1c GA levels were measured in blood samples collected from 307 diabetic subjects of whom 258 were on HD and 49 were without overt renal disease. The results showed that in diabetic HD patients, HbA1c levels significantly underestimate glycemic control while those of GA more accurately reflect this control. ${ }^{10}$

In a study by Freedman et al. (USA, 2010) HbA1c and GA\% were measured in 519 diabetic subjects: 55 on peritoneal dialysis (PD), 415 on HD, and 49 non-nephropathy controls. They concluded that the relationship between HbA1c and GA\% differs in diabetic patients with ESRD who perform either PD or HD compared to those without nephropathy. HbA1c significantly underestimated glycemiccontrol in PD and HD patients relative to GA\%. ${ }^{12}$

In a study by Dawlat Sany et al ( Saudi , 2013) which was composed of $25 \mathrm{HD}$ patients with type2 diabetes, $25 \mathrm{HD}$ patients without diabetes, 25 patients with type- 2 diabetes and chronic kidney disease(CKD) and 10 patients with type- 2 diabetes and normal renal function, they concluded that GA provides a significantly better measure to estimate glycemic control in HD patients with diabetes and that the assessment of glycemic control by $\mathrm{HbA} 1 \mathrm{c}$ in these patients might lead to likely underestimation as a result of the increasing proportion of young erythrocyte by the use of erythropoietin. ${ }^{13}$

In a study by Freedman et al (USA, 2011) Quarterly GA levels were measured for up to 2.33 years in 444 prevalent patients with diabetes and ESRD. Proportional hazard time-dependent covariate models were computed with adjustment for demographic characteristics, comorbidities, and laboratory variables. Similar analyses were performed for available HbA1c and monthly random serum glucose determinations.

There were 156 deaths during the observation period. HbAlc and random serum glucose concentrations were not predictive of survival. Increasing GA levels were associated with hospitalization in the 17 days after measurement, whereas HbA1c was not. Hence, they concluded thatIn contrast to the $\mathrm{HbA} 1 \mathrm{c}$ and random serum glucose values, GA accurately predicts the risk of death and hospitalizations in patients with diabetes mellitus and ESRD. ${ }^{14}$ 


\section{Methodology}

This is a comparative study which included 100 patients who fulfilled the inclusion and exclusion criteria in the out-patient and in-patient Department of General Medicine, Kempegowda Institute of Medical Sciences, Bangalore between January 2016to June 2016.

\section{Inclusion Criteria}

Sample Size -100 patients

Cases- 50 diabetic ESRD patients on haemodialysis, above 30 years of age.

Controls - 50 diabetic patients with normal renal function (serum creatinine $<1.2 \mathrm{mg} / \mathrm{dl}$ ) above 30 years of age.

\section{Exclusion Criteria}

Patients with: hemoglobinopathy, anemia due to causes other than CKD, hepatic disorders, inflammatory conditions, thyroid disease, heavy proteinuria

\section{Methods of Data Collection}

Informed consent was taken from all patients included in the study. A pre-structured and pretested pro forma was used to collect the data. Baseline data including demographics and detailed medical history including duration of diabetes, treatment and other co-morbidities was taken. In subjects on dialysis, the duration of dialysis, the history of the doses of erythropoietin, and/or receiving blood transfusion over the preceding 3 months was taken. A thorough clinical examination was done including Direct Ophthalmoscopy to assess retinopathy. Patients either provided all the latest reports of relevant laboratory investigations done within the prior month (including FBS, PPBS, CBC, S. albumin, renal function tests and urine routine) or the investigations were done during the study.

$10 \mathrm{ml}$ of venous blood and urine samples were collected from all patients. Blood was drawn from the dialyzer circuit in subjects with ESRD, prior to initiation of dialysis or administration of anticoagulants. Blood samples were then divided with $5 \mathrm{ml}$ sent for HbA1c in EDTA tube. $5 \mathrm{ml}$ collected in plain tube and the serum was allowed to clot for 10-20 minutes. Then centrifuged ( at 2000 RPM) for 20 minutes. The supernatant was collected and stored at -20 degree celsius for future measurement of GA. Egfr was measured using the MDRD formula.

HbA1c was analyzed within $24 \mathrm{~h}$ of collection (refrigerated if not immediately assayed) on a cation exchange column chromatograph using an automated high-pressure liquid chromatography instrument.

For the estimation of GA, the Human Glycated Albumin ELISA kit which was provided by the Bioassay Technology Laboratory (China) was used. The kit uses enzyme-linked immunosorbent assay (ELISA) based on the biotin double antibody sandwich technology to assay the level of GA in samples. The serum samples were added to monoclonal antibody Enzyme wells which is pre-coated with Human GA monoclonal antibody and incubated. Then GA antibodies labeled with biotin was combined with Streptavidin-HRP to form immune complex which was then incubated and washed again to remove the uncombined enzyme. Then Chromogen Solution A,B, were added and the color of the liquid changes. The chroma of color and the concentration of the Human Substance Glycated Albumin (GA)of sample were positively correlated. The optical density (OD) values of each sample was registered. The standard curve was drawn on a graph with the concentration of standards as the abscissa and the OD value as the ordinate. According to OD value, the corresponding concentration was estimated as the GA value.

Assay range $-0.5 \mathrm{ng} / \mathrm{ml}-200 \mathrm{ng} / \mathrm{ml}$

Sensitivity $-0.24 \mathrm{ng} / \mathrm{ml}$

$<50 \mathrm{ng} / \mathrm{ml}$ reflects good glycemic control $50-100 \mathrm{ng} / \mathrm{ml}$ reflects average glycemic control $>100 \mathrm{ng} / \mathrm{ml}$ reflects poor glycemic control

Statistical Analysis: Proportions were compared using Chi-square test of significance. The student ' $t$ ' test was used to determine whether 


\section{JMSCR Vol||05||Issue||12||Page 31717-31725||December}

there was a statistical difference between the groups in the parameters measured. Pearson correlation coefficients were calculated to determine whether there was any correlation between the parameters measured. Data analysis was carried out using Statistical Package for Social Science (SPSS ver 10.5) package.

\section{Results}

The mean age of controls and cases were $54.56 \pm$ 8.1 year and $57.92 \pm 10.54$ years respectively $(\mathrm{p}$ 0.077). The number of males and females in the control group was 26 and 24 respectively, whereas in the case group there were 42 males and 8 females(p-0.001)

The mean BMI of controls was 24.3 and that of cases was 23.4(p-0.002)

Among the controls and cases, the mean duration of diabetes was $3.68 \pm 1.67$ years and $11.72 \pm 2.6$ years respectively $(\mathrm{p}<0.001) .45$ out of 50 controls were on Oral Hypoglycemic agents alone and the remaining 5 were on OHAs and insulin both. All 50 cases were on insulin. $16 \%$ of controls and $30 \%$ of cases were hypertensive
patients.As part of treatment of anemia ,in the preceding 3 months ,25 cases received packed cell transfusion and 14 cases received erythropoietin injections.

$\mathrm{Hb}$ and S.Albumin levels were significantly higher in controls $(12.4 \pm 0.8$ and $4.10 \pm 0.1$ respectively) than cases $(9.8 \pm 0.6$ and $3.46 \pm 0.2$ respectively). Whereas Creatinine levels were higher in cases $(4.99 \pm 0.7)$ than controls $(0.9 \pm 0.1)$. FBS, PPBS and GA in controls was $187 \pm 57.8 \mathrm{mg} / \mathrm{dl}, 231.7 \pm 73.7 \mathrm{mg} / \mathrm{dl}, 72.84 \pm 32.42 \mathrm{ng}$ $/ \mathrm{ml}$ and in cases was $184 \pm 53.1 \mathrm{mg} / \mathrm{dl}, 224.7 \pm 72.5$ $\mathrm{mg} / \mathrm{dl}, \quad 75.20 \pm 34.7 \mathrm{ng} / \mathrm{ml}$ respectively. The difference of FBS, PPBS and GA between controls and cases was not statistically significant. However, HbA1c was significantly lower in cases $(7.08 \pm 1.2)$ compared to controls $(9.26 \pm 2.01)$

The HbA1c levels were significantly and paradoxically lower in diabetic patients with end stage renal disease on haemodialysis compared to diabetics with normal renal function. GA better reflected the glycemic control in these patients.

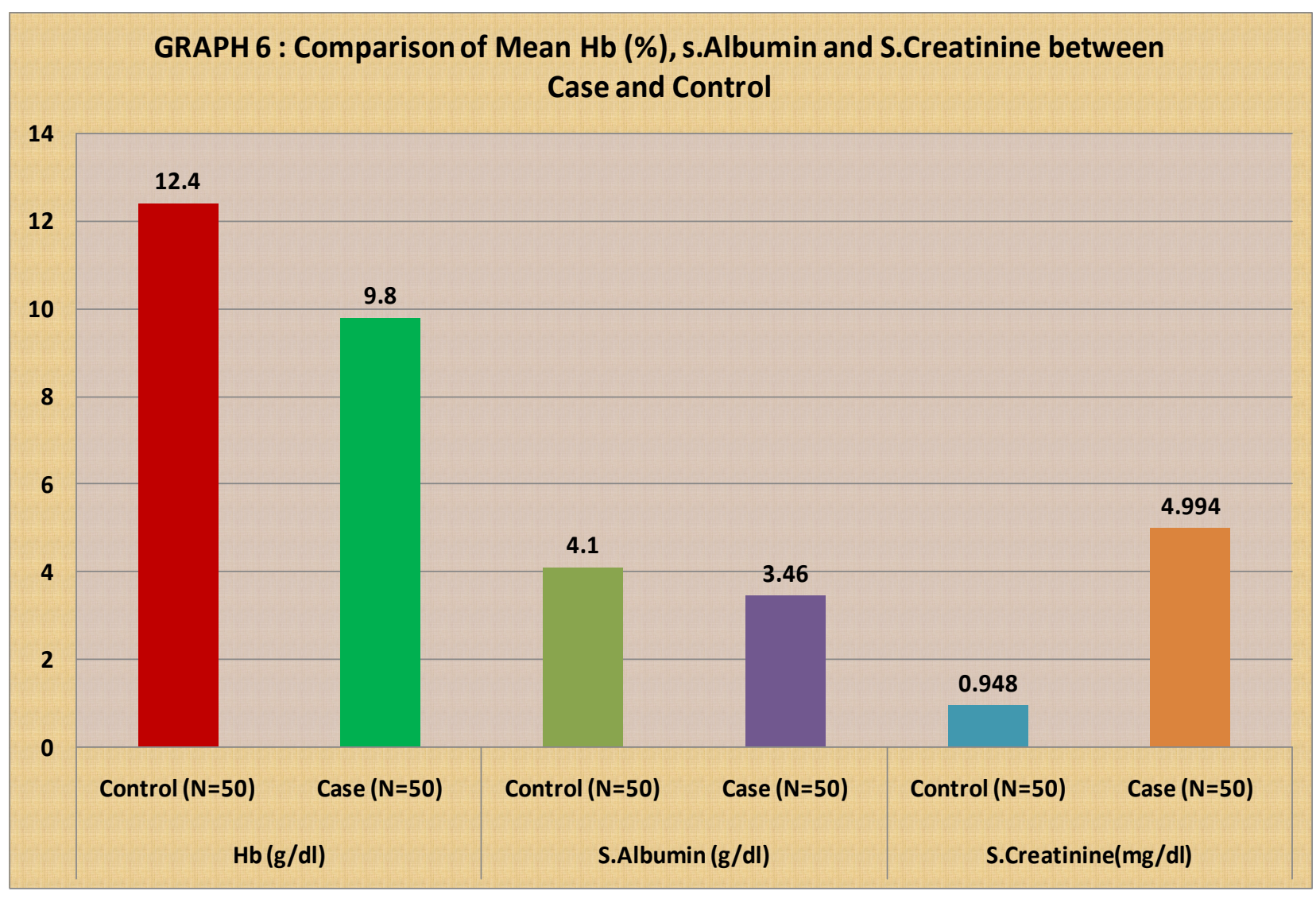




\section{JMSCR Vol||05||Issue||12||Page 31717-31725||December}
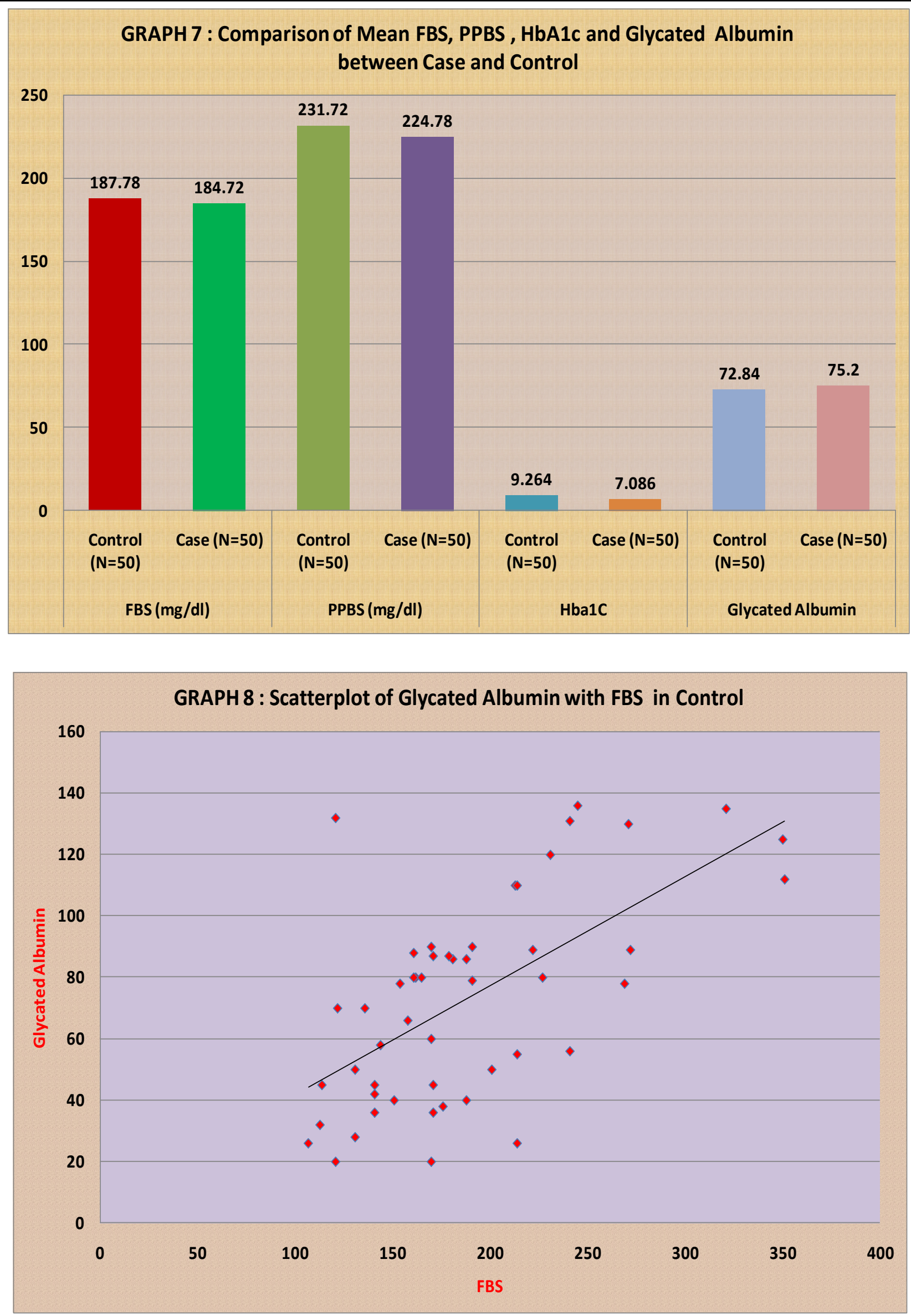


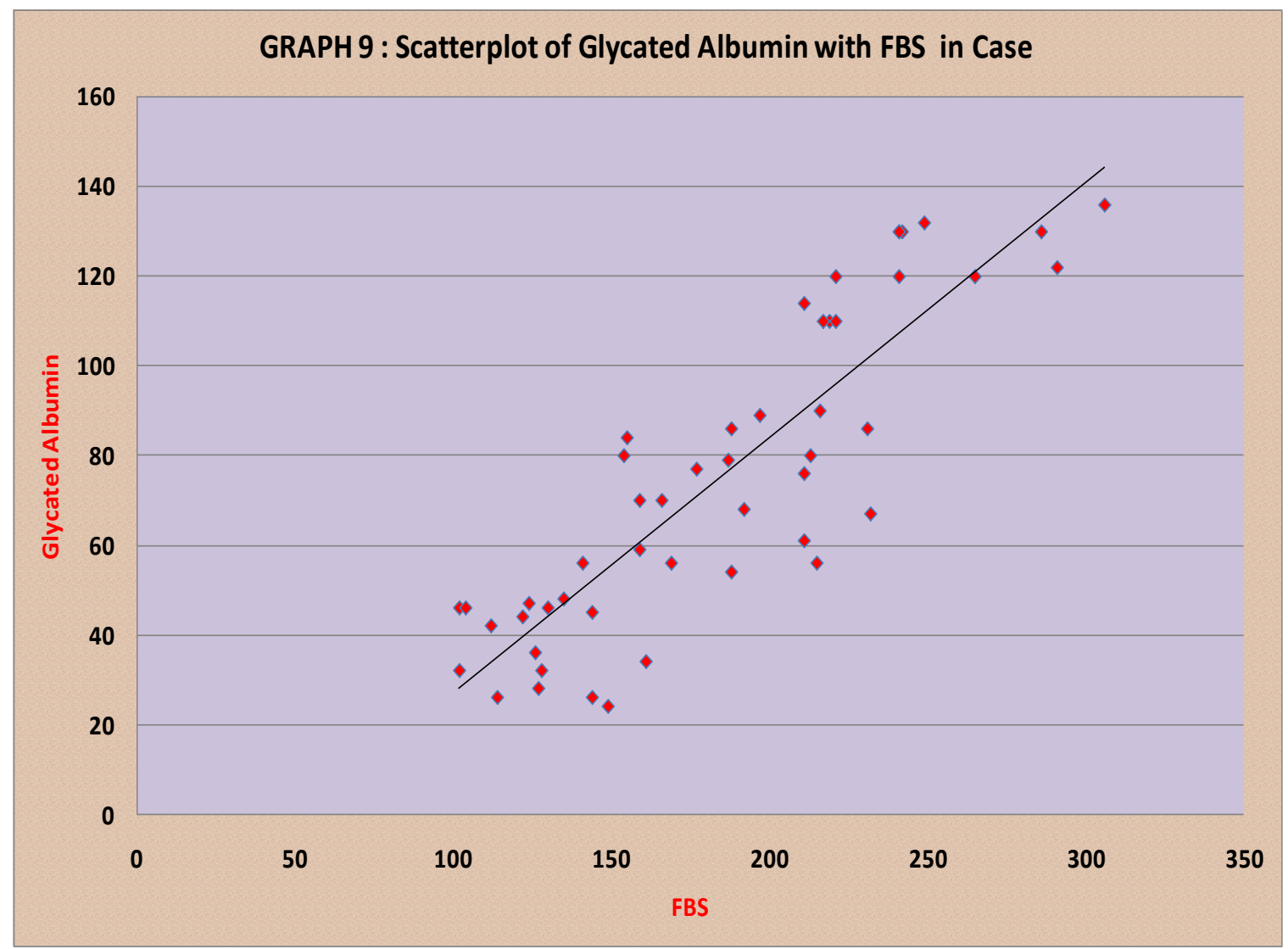

\section{Discussion}

In the present study, there was no statistical difference between FBS and PPBS in the control and case study groups. However, the HbA1C levels was drastically lower and GA levels were higher in the cases compared to the control group. The results were similar to the studies done by Peacock et $\mathrm{al}^{10}$ and Freedman et $\mathrm{al}^{12}$ wherein the HbA1C was paradoxically lower among the cases compared to controls. The GA levels were also higher in the cases than controls, in these studies.

In the study by Freedman et $\mathrm{al}^{12}$, Diabetic controls without kidney failure had mean serum creatinine concentration $1.0 \pm 0.3 \mathrm{mg} / \mathrm{dL}$. The mean $\pm \mathrm{SD}$ of the last three monthly serum glucose concentrations in diabetic HD $(169.7 \pm 62 \mathrm{mg} / \mathrm{dL})$ was higher than in non-nephropathy controls $(146.1 \pm 66 \mathrm{mg} / \mathrm{dL} ; \mathrm{p}=0.03)$. In the present study the the diabetic controls had mean serum creatinine of $0.97 \pm 0.1 \mathrm{mg} / \mathrm{dl}$.FBS and PPBS in controls was $187 \pm 57.8 \mathrm{mg} / \mathrm{dl}$ and $231.7 \pm 73.7 \mathrm{mg} /$ $\mathrm{dl}$, and in cases was $184 \pm 53.1 \mathrm{mg} / \mathrm{dl}$ and $224.7 \pm 72.5 \mathrm{mg} / \mathrm{dl}$ respectively which was higher in controls.
Inaba et $\mathrm{al}^{11}$,studied the Effect of a Single HD Session on GA and HbA1c and determined that Serum GA values were almost identical between before and after a single HD session in HD patients $\left(r_{-} 0.998, P_{-} 0.001\right)$; serum HbA1c also correlated significantly in a positive manner $(r$ $0.992, P_{-}$0.001) but to a lesser degree. These data clearly indicated that the substances that accumulated into uremicserum did not affect GA values at all. In the present study such an observation was not attempted.

Inaba et al also studied the Correlation between GA and Serum Albumin and between HbA1c and Hemoglobin Levels in HD Patients with Diabetes and found significant and negative correlation was found between GA and serum albumin levels $(r$ _0.131, $P$ _ 0.002; , although HbA1c did not correlate with serum albumin levels $\left(r_{-} 0.010, P\right.$ _ 0.853). In contrast, there was a significant and positive correlation between HbA1c and haemoglobin levels $\left(r \_0.090, P_{-}\right.$0.036; although GA did not correlate with serum $\mathrm{Hb}$ levels $\left(r_{-} 0.037, P_{-} 0.397\right)$. The present study also reflected similar findings. 
The GA value in our study differed from those used in the other studies, as the GA estimation was done using different assays. The other studies took the RBS of preceding 3 months into consideration to estimate average plasma glucose. In the present study we used FBS and PPBS levels which is theoretically more accurate.

It is clear that the HbAlc assay has severe limitations in diabetic patients on HD, markedly underestimating recent glycemic control compared to serum glucose concentrations and GA. This report extends the findings of the accuracy of the HbA1c assay in HD and to demonstrate that HbA1c is significantly impacted by ESRD.

In the study by Freedman et $\mathrm{al}^{12}$, where they also evaluated patients on PD, they made the important observation that $\mathrm{HbA} 1 \mathrm{c}$ readings are falsely low in patients on either form of dialysis, $\mathrm{HD}$ and $\mathrm{PD}$, which calls into question the accuracy of the HbA1c assay in diabetic patients with severely reduced renal function, and potentially those not yet on RRT.

It is highly likely that the frequent coexistence of low $\mathrm{Hb}$ concentrations and higher doses of erythropoietin, both having profound effects on the HbA1c assay, are related to shortened RBC survival. This leads to reduced time for glucose and haemoglobin to interact and form glycosylated hemoglobin.

This makes it critical that the relationship between serum glucose concentrations and GA and HbA1c assays be compared in patients with stages III - V CKD to determine whether clinicians and patients are basing diabetes therapy on falsely low HbA1c results. If $\mathrm{HbA1c}$ is falsely low in CKD, this could be placing diabetic CKD patients at risk for more rapid progression of nephropathy to ESRD, and speeding the progression of other microvascular (and possibly macrovascular) complications.

The nonenzymaticglycation of various proteins is increased in patients with diabetes as a result of sustained higher PG and The rate of production also depends on the half-life of each protein. HbAlc provides an integrated measure of $P G$ during the previous 2 to 3 mo as a result of the long life span of erythrocytes (120 days), whereas GA has been hypothesized to be a glycemic indicator during the immediately previous 2 weeks. Although a rapid change in glycemic control may reflect a greater change of GA than HbA1c, this study examined the significance of GA compared with HbA1c under stationary state of diabetic control, without any change of antidiabetic drugs during the study period, and compared GA and HbA1c values in patients with diabetes and with and without renal dysfunction.

Theoretically, proteinuria can impact GA\% based upon reduced exposure of serum albumin to glucose. Freedman et al ${ }^{12}$ addressed this issue by comparing GA\%/HbA1c in 15 anuric diabetic patients lacking proteinuria (mean \pm SD $2.71 \pm$ 0.63 , median 2.58) with that in 12 diabetic patients with urine output exceeding $1 \mathrm{~L}$ per day $(2.81 \pm 0.68$, median 2.92). Significant differences were not seen in $\mathrm{GA} \% / \mathrm{HbA} 1 \mathrm{c}$ between these groups $(\mathrm{p}=0.49)$, suggesting that proteinuria did not contribute to the results. The present study also showed a negative correlation coefficient between GA and S. albumin, indicating that there is no positive relationship between these indices.

Concerns exist regarding the accuracy of this test in diabetic subjects with advanced

CKD not yet on dialysis. Comparisons of simultaneous GA and $\mathrm{HbA1c}$ assay results in CKD patients not yet on dialysis are underway. The results of those studies will determine whether the HbA1c assay should play a role in diabetic patients with advanced CKD or ESRD.

GA acquires biologic properties that are linked to the pathogenesis of diabetic vascular complications, suggesting that GA not only is significant as an indicator of hyperglycemia but also contributes directly to vascular injury. As such, GA is better than $\mathrm{HbA} 1 \mathrm{c}$ in predicting the development of vascular complications in HD patients with diabetes. 


\section{Conclusion}

The HbA1c levels were significantly and paradoxically lower in diabetic patients with end stage renal disease on haemodialysis compared to diabetics with normal renal function. Glycated albumin better reflected the glycemic control in these patients.

Accurate determination of glycemic control is of paramount importance in the diabetic population, as improved glycemic control reduces micro- and macrovascular complications in patients with type 1 and type 2 diabetes mellitus. Diabetic dialysis patients are especially at high risk, as they succumb to cardiovascular and infectious complications during their first year of renal replacement therapy.

Glycemic control improves survival in patients on hemodialysis. Moreover, mild degrees of hyperglycemia in non diabetic dialysis populations have been associated with reduced survival. An accurate assessment of glycemic control in the dialysis population is therefore critical, to improve outcomes and survival.

$\mathrm{HbA} 1 \mathrm{c}$ has been a cornerstone in the evaluation of diabetic patients. This measurement relies on a relatively stable RBC survival, but in patients on hemodialysis, shortened RBC survival, red cell transfusions and receiving erythropoietin are likely to lower the $\mathrm{HbA1c}$, potentially making it unreliable in assessing glycemic control. Hence, Glycated Albumin estimation should be considered in these patients to determine the glycemic control more accurately.

\section{Limitations}

The sample size in the study is small. A larger sample size would be needed to better achieve the aim of this study.

\section{References}

1. The Diabetes Control and Complications Trial Research Group: The effect of intensive treatment of diabetes on the development and progression of long-term complications in insulin-dependent diabetes mellitus. N Engl J Med 329: $977-$ 986, 1993

2. The DCCT Research Group: Diabetes Control and Complications Trial (DCCT): Results of feasibility study. Diabetes Care 10: 1-19, 1987

3. Ohkubo Y, Kishikawa H, Araki E, Miyata $\mathrm{T}$, Isami S, Motoyoshi S, Kojima Y, Furuyoshi N, Shichiri M: Intensive insulin therapy prevents the progression of diabetic microvascular complications in Japanese patients with non-insulindependent diabetes mellitus: A randomized prospective 6-year study. Diabetes Res Clin Pract 28: 103-117, 1995

4. UK Prospective Diabetes Study (UKPDS) Group: Intensive blood-glucose control with sulphonylureas or insulin compared with conventional treatment and risk of complications in patients with type 2 diabetes (UKPDS 33). Lancet 352: 837853,1998

5. Cao JJ, Hudson M, Jankowski M, Whitehouse F, Weaver WD: Relation of chronic and acute glycemic control on mortality in acute myocardial infarction with diabetes mellitus. Am J Cardiol 96: 183-186, 2005

6. Gaede P, Vedel P, Larsen N, Jensen GV, Parving $\mathrm{HH}$, Pedersen $\mathrm{O}$ : Multifactorial intervention and cardiovascular disease in patients with type 2 diabetes. N Engl J Med 348: 383-393, 2003

7. Calisti L, Tognetti S. Measure of glycosylated hemoglobin. Acta Biomed 2005; 76(Suppl 3): 59-62.

8. Goldstein DE, Little RR, Lorenz RA et al. Tests of glycemia in diabetes. Diabetes Care 2004; 27: 1761-1773

9. Ly J, Marticorena R, Donnelly S. Red blood cell survival in chronic renal failure. Am J Kidney Dis 2004; 44: 715-719.

10. Peacock TP, Shihabi ZK, Bleyer AJ, Dolbare EL, Byers JR, Knovich MA, et al. Comparison of glycated albumin and 
hemoglobinA1c levels in diabetic subjects on hemodialysis. Kidney Int 2008; 73:1062-8; epub 20 Feb 2008.

11. Inaba M, Okuno S, Kumeda Y, Yamada S, Imanishi $\mathrm{Y}$, Tabata $\mathrm{T}$, et al. Glycated albumin is a better glycemic indicator than glycatedhemoglobin values in hemodialysis patients with diabetes: effect of anemia and erythropoietin injection. $\mathrm{J}$ Am SocNephrol 2007; 18:896-903

12. Barry I. Freedman, Rajeev N. Shenoy,Jonathan A. Planer,Kimberly D. Clay, Zak K. Shihabi, et al Comparison Of Glycated Albumin And Hemoglobin A1c Concentrations In Diabetic Subjects On Peritoneal And Hemodialysis Peritoneal Dialysis International, 2010 Vol. 30, pp. 72-79,

13. DawlatSany, Yasser Elshahawy, Walid Anwar Glycated Albumin versus Glycated Hemoglobin as Glycemic Indicator in Hemodialysis Patients with Diabetes Mellitus: Variables that Influence Saudi J Kidney Dis Transpl 2013;24(2):260-273

14. Barry I. Freedman,* LilianAndries, ${ }^{*}$ Zak K. Shihabi et al Glycated Albumin and Risk of Death and Hospitalizations in Diabetic Dialysis Patients Clin J Am SocNephrol 6: 1635-1643, 2011. 